\title{
Caracterização química, mecânica e morfológica do gesso $\beta$ obtido do pólo do Araripe
}

\section{(Chemical, mechanical and morphological characterization of gypsum obtained at Araripe, PE, Brazil)}

\author{
A. A. Barbosa ${ }^{1,2}$, A. V. Ferraz ${ }^{1,2}$, G. A. Santos ${ }^{2}$ \\ ${ }^{1}$ Grupo de Pesquisa em Materiais - GPEM, Instituto de Pesquisa em Ciência dos Materiais - IPCM \\ ${ }^{2}$ Universidade Federal do Vale do S. Francisco, Campus Juazeiro, BA, Brasil \\ amanda.barbosa@univasf.edu.br
}

\begin{abstract}
Resumo
$\mathrm{O}$ gesso é um sulfato de cálcio hemihidratado com fórmula química $\mathrm{CaSO}_{4} \cdot 0,5 \mathrm{H}_{2} \mathrm{O}$, este é produzido em grande abundância na região do Araripe estado de Pernambuco e apresenta uma pureza que varia de 80 a $95 \%$, sua aplicação baseia-se principalmente na construção civil e em um segundo plano observa-se sua utilização na odontologia, industria química, ortopedia etc. Frente à elevada qualidade da matéria prima em questão e ao baixo emprego do gesso em materiais e objetos de maior valor agregado, verificouse a necessidade da caracterização desta matéria prima para um maior conhecimento de suas propriedades, sendo realizadas para tal, análises de FTIR, DRX, FRX, MEV com EDS acoplado, ensaios mecânicos de resistência à compressão e à flexão, cálculo da porosidade geométrica dos corpos sólidos, análise granulométrica das partículas e avaliação do tempo de pega da pasta. Este estudo tem a finalidade de conhecer detalhadamente suas características químicas, morfológicas e mecânicas, para que então posteriormente tais informações possam ser válidas para estudos referentes ao melhoramento de suas propriedades visando sua aplicação em novos produtos.
\end{abstract}

Palavras-chave: gesso, caracterização, propriedades.

\begin{abstract}
Gypsum is a calcium sulfate hemihydrate with chemical formula $\mathrm{CaSO}_{4} .0 .5 \mathrm{H}_{2} \mathrm{O}$, largely produced in Araripe, state of Pernambuco, Brazil, and features a purity ranging from 80 to 95\%; their implementation is mainly based on the construction and also in dentistry, chemical industry, orthopedics, etc.. Due to the high quality of raw material and the low use of gypsum materials and objects with higher added value, there was the need to characterize the raw material for a better knowledge of its properties. FTIR, XRD, XRF, SEM, compressive and flexural strength, evaluation of porosity, particle size analysis and evaluation of the setting time of the paste procedures were performed. This study aims to know in detail the chemical, morphological and mechanical characteristics of gypsum, to be able to improve its properties for application in new products.
\end{abstract}

Keywords: gypsum, characterization, properties.

\section{INTRODUÇÃO}

Atualmente, estudos encontrados na literatura demonstram que o gesso é um material muito empregado e estudado no mundo inteiro. $\mathrm{O}$ gesso consiste no sulfato de cálcio hemihidratado, com fórmula química $\mathrm{CaSO}_{4} \cdot 0,5 \mathrm{H}_{2} \mathrm{O}$. A produção de gesso natural acontece basicamente em quatro etapas: extração do gipso; preparação para calcinação; calcinação e seleção. O gipso é uma rocha sedimentar que apresenta basicamente em sua composição a gipsita, a anidrita e algumas impurezas, geralmente argilominerais, calcita, dolomita e material orgânico. A gipsita é o mineral compacto de baixa dureza, pouco solúvel em água, que é a matéria prima para o gesso; sua fórmula química é $\mathrm{CaSO}_{4} \cdot 2 \mathrm{H}_{2} \mathrm{O}$ [1]. Após a extração, a gipsita passa por alguns processos de beneficiamento para adequação ao tipo de forno onde será calcinada. Basicamente, as etapas são as seguintes: britagem, moagem grossa; estocagem; secagem; moagem fina e acondicionamento. A calcinação é o processo térmico pelo qual a gipsita é desidratada. $\mathrm{O}$ material é calcinado numa faixa de temperatura de $140{ }^{\circ} \mathrm{C}$ a $160{ }^{\circ} \mathrm{C}$, para que $75 \%$ da água de cristalização seja retirada da estrutura para obter o hemidrato $\left(\mathrm{CaSO}_{4} \cdot 1 / 2 \mathrm{H}_{2} \mathrm{O}\right)$, conforme mostrado na equação $\mathrm{A}[1]$.

$\mathrm{CaSO}_{4} \cdot 2 \mathrm{H}_{2} \mathrm{O}+23,81 \mathrm{cal} / \mathrm{g} \rightarrow \mathrm{CaSO}_{4} \cdot 1 / 2 \mathrm{H}_{2} \mathrm{O}+3 / 2 \mathrm{H}_{2} \mathrm{O}$

A calcinação pode ser por via seca ou úmida. Se o gipso for calcinado a seco sob pressão atmosférica, ou baixa pressão, será obtido o hemidrato $\beta$. Caso a calcinação ocorra sob pressão de vapor de água saturante, será obtido o hemidrato $\alpha$ [1]. O gesso $\alpha$, devido ao seu processo de produção, 
apresenta aplicações mais nobres (gesso hospitalar) e conseqüentemente alcança preços mais elevados. $\mathrm{O}$ gesso $\beta$, com custo de produção mais baixo, predomina no gesso de construção nacional.

A escolha do estudo focado no gesso $\beta$ é devido à grande abundância de jazidas de gipsita na região do pólo do Araripe e ao simples processo para a fabricação do gesso $\beta$, com baixo custo de produção. Juntando-se a isto, sua característica de fácil recristalização pela adição de água torna-o bastante atrativo para a indústria, que o emprega em variados produtos [2]. A fonte da qual se retira todo o gesso empregado na indústria, está localizada especialmente na região da Bacia Sedimentar do Araripe, que abrange os estados de Pernambuco, Piauí e Ceará [1]. No denominado pólo gesseiro do Araripe formado pelos Municípios de Araripina, Bodocó, Ipubi, Ouricuri e Trindade, todos no estado de Pernambuco, concentram-se 95\% da produção brasileira de gesso. A elevada demanda de gesso na região atribui-se as boas condições de mineração das jazidas, além do fato destas apresentarem um minério de excelente qualidade [3] com pureza que varia de 80 a 95\% [4]. Todo o gesso gerado a partir da extração da gipsita, na região do Araripe, encontra aplicação em diversas atividades industriais como, por exemplo: gesso para a construção civil e gesso industrial. Do gesso natural obtido através da calcinação da gipsita, cerca de 96\% destina-se diretamente ao emprego na construção civil. Este se apresenta de duas formas, o gesso de fundição e o de revestimento, os quais são utilizados na confecção de placas, blocos e revestimentos de paredes. O gesso industrial trata-se de um produto de maior pureza, é utilizado na confecção de cerâmicas, porcelanas e também como fonte de cálcio e enxofre na indústria do vidro. Outras aplicações de menores proporções são observadas, tais como: carga mineral na fabricação de papéis, plásticos e adesivos; condicionadores de solo; indústria ortopédica e odontológica. Como se pode constatar, o gesso $\beta$ recebe muitas aplicações, no entanto, ao se observar o seu emprego na indústria em termos de proporções percentuais, Tabela I, verifica-se que em geral o gesso $\beta$ é utilizado prioritariamente na construção civil [1].

Tabela I - Principais usos comerciais do gesso no Brasil. [Table I - Main commercial uses of gypsum in Brazil.]

\begin{tabular}{cc}
\hline Aplicação & Consumo \\
\hline Pré-moldado & $61 \%$ \\
Revestimento & $35 \%$ \\
Moldes cerâmicos & $3 \%$ \\
Outros & $1 \%$ \\
\hline
\end{tabular}

Esta realidade mostra que apesar da alta qualidade do minério e da grande abundância deste na região, suas propriedades não estão sendo exploradas devidamente, visto que apesar da importância do gesso na construção civil, o mesmo possui um baixo valor agregado. Por outro lado, verifica-se que uma substância química tão pura, como o sulfato de cálcio hemihidratado produzido na região do
Araripe [4], apresenta um grande potencial que deve ser estudado para o desenvolvimento de materiais e produtos mais nobres. Um exemplo de estudos para a valorização desta matéria prima pode ser constatado na literatura, onde se observa a síntese química de um material que possui interesse para aplicações em implantes médicos, a hidroxiapatita, e, portanto, apresenta um alto valor agregado, utilizando como principal material de partida em sua síntese, o gesso $\beta$ [5].

Este trabalho tem como objetivo caracterizar o hemidrato e dihidrato do gesso proveniente do polo gesseiro do Araripe. $\mathrm{O}$ estudo tem a finalidade de conhecer detalhadamente suas características químicas, morfológicas e mecânicas, para que então posteriormente tais informações possam ser válidas para estudos referentes ao melhoramento de suas propriedades visando sua aplicação em novos produtos.

\section{MATERIAIS E MÉTODOS}

\author{
Caracterização do gesso $\beta\left(\mathrm{CaSO}_{4} \cdot 0,5 \mathrm{H}_{2} \mathrm{O} /\right.$ sulfato de \\ cálcio hemihidratado)
}

A primeira etapa no desenvolvimento experimental consistiu em caracterizar o gesso $\beta$. O gesso utilizado foi fornecido pela Indústria Gesso Mineral Ltda., fábrica pertencente ao Pólo Gesseiro do Araripe. O hemihidrato em pó foi caracterizado originalmente como saiu de fábrica. A identificação das fases cristalinas presentes foram determinadas por difração de raios X (DRX), com o equipamento Difract ACT série 1000 (Siemens), utilizando a linha $\mathrm{k} \alpha$ do cobre $(\lambda=1,54056 \AA)$ a $40 \mathrm{kV}$ e $40 \mathrm{~mA}$. A aquisição foi no intervalo de $2 \theta$ de $5^{\circ}$ a $120^{\circ}$, com um passo de $0,02^{\circ}$ e tempo de integração de 1 s por ponto. As fases foram identificadas com os dados dos arquivos JCPDS.

A avaliação morfológica foi feita em um microscópio eletrônico de varredura (Hitachi, TM-1000), acoplado a um microanalisador EDS (espectroscopia de dispersão de energia por raios X), para identificação dos elementos constituintes e eventuais impurezas.

A composição química da amostra foi determinada pela técnica de fluorescência de raios X (FRX) (EDX900).

Os espectros de transmissão na região do infravermelho (4000 a $400 \mathrm{~cm}^{-1}$ ) foram registrados em um espectrômetro com transformada de Fourier (FTIR) Bruker I FS66. O composto analisado foi preparado em forma de pastilhas de $\mathrm{KBr}$ prensadas sob vácuo.

Acaracterização do gesso foi realizada por granulométrica a laser, realizada com o Mastersizer 2000 - Malvern Instr. Ltd. Versão 5.1 13/6/01, software de versão 5.1, que mede partículas de diâmetros de 0,02 a $2000 \mu$.

Caracterização do corpo sólido de gesso $\beta\left(\mathrm{CaSO}_{4} \cdot 2 \mathrm{H}_{2} \mathrm{O} /\right.$ sulfato de cálcio dihidratado)

A primeira etapa no desenvolvimento experimental foi caracterizar o gesso $\beta$ (sulfato de cálcio hemidratado). Após a caracterização, a pasta do corpo sólido de gesso foi preparada conforme proposto pela NBR 12128 (ABNT, 
1991) [6]: polvilhamento do pó de gesso sobre a água durante $1 \mathrm{~min}$; repouso da suspensão por $2 \mathrm{~min}$; e mistura da pasta durante $1 \mathrm{~min}$. Logo após a mistura das pastas, os moldes foram preenchidos lentamente, em camadas, para evitar o aprisionamento do ar na pasta durante a operação. A compactação de cada camada foi efetuada manualmente com golpes desferidos com espátula. Cerca de $2 \mathrm{~h}$ (dependendo da razão em massa de água e do gesso presentes na amostra) após a moldagem, os corpos de prova foram retirados dos moldes. Os fatores considerados foram razão em massa água/gesso $(\mathrm{a} / \mathrm{g}=0,5 ; 0,6$ e 0,7$)$. As variáveis avaliadas foram tempo de pega e resistência mecânica a compressão e flexão. A determinação do tempo de pega foi realizado pelo método da espátula proposto pela norma alemã DIN 1168

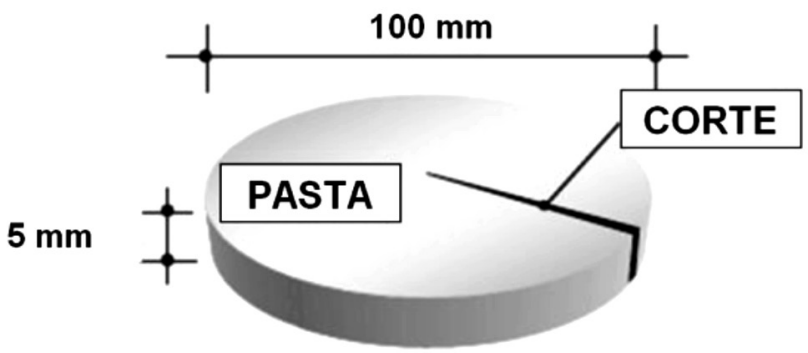

Figura 1: Esquema para avaliação de início e fim de pega, segundo a norma DIN 1168.

[Figure 1: Scheme for evaluating beginning and end of handle, DIN 1168.]
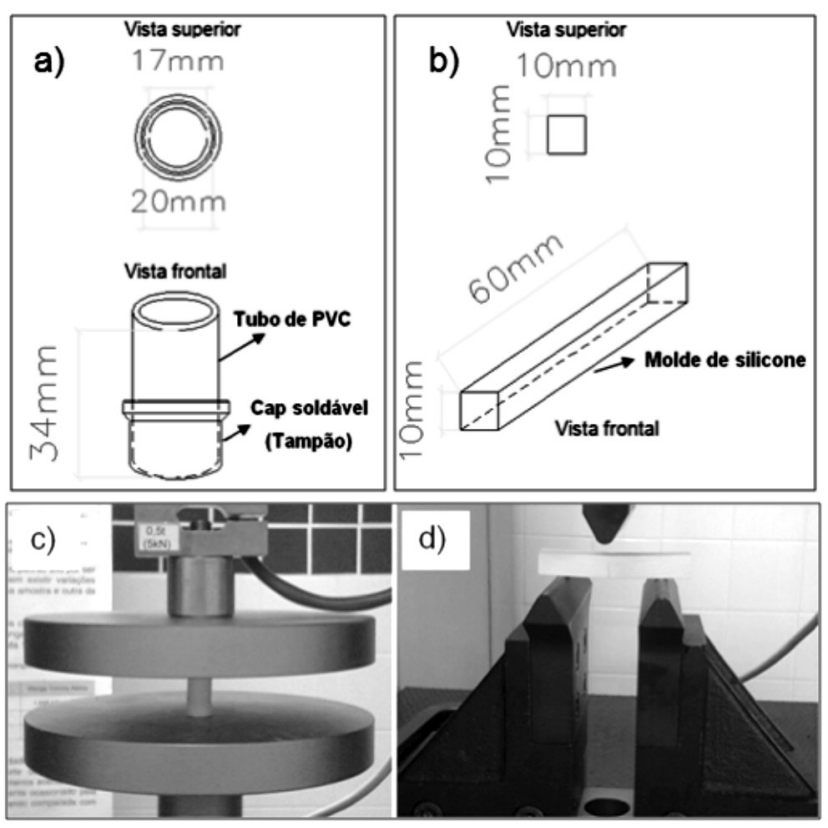

Figura 2: a) Desenho do molde cilíndrico; b) Desenho do molde retangular; c) Corpo de prova cilíndrico de gesso sob ensaio de $\boldsymbol{\sigma c}$ e d) Corpo de prova retangular de gesso sob ensaio de $\boldsymbol{\sigma} \mathbf{f}$.

[Figure 2: a) Drawing of the cylindrical mold; b) Drawing of rectangular mold; c) Cylindrical specimen of plaster under test $\boldsymbol{\sigma} c$ and d) Rectangular plaster specimen under $\boldsymbol{\sigma}$ f test.]
[7]. Esse método consiste em moldar um corpo de prova com 5,0 $\mathrm{mm}$ de espessura e $100 \mathrm{~mm}$ de diâmetro, mostrada na Fig. 1.

O início de pega é o momento em que o corte aberto pela espátula não mais se fecha. O final de pega é determinado como o momento em que se pressiona o dedo sobre o corpo de prova, se este deixar impressão na pasta, a pega ainda não foi concluída, isto é, o final da pega é determinado pelo momento em que o dedo não mais deixa impressão sobre a pasta [7]. Seguindo o mesmo procedimento de preparo da pasta e utilizando-se razões a/g de 0,$5 ; 0,6$ e 0,7 , foram obtidos corpos de prova para a caracterização mecânica do material. Os corpos foram formados pela adição da pasta em moldes cilíndricos de PVC com dimensões $(\mathrm{h}=34 \mathrm{~mm} \times \Phi$ $=17 \mathrm{~mm}$ ) para ensaios de resistência à compressão $(\boldsymbol{\sigma c}) \mathrm{e}$ moldes retangulares de silicone com dimensões $(\mathrm{a}=10 \mathrm{~mm}$ $\mathrm{x} \mathrm{b}=10 \mathrm{~mm} \mathrm{x} \mathrm{c}=60 \mathrm{~mm}$ ) para ensaios de resistência à flexão ( $\boldsymbol{\sigma f})$, como mostrado na Fig. 2. Os ensaios foram realizados em uma máquina universal de ensaios mecânicos (Emic-DL 10000). A porosidade geométrica dos corpos foi calculada pela equação (B) [8].

$$
\varepsilon(\%)=1-\left(\varphi_{\mathrm{g}} \varphi_{\mathrm{s}}\right) \times 100
$$

na qual $\varepsilon$ - porosidade, $\varphi_{\mathrm{g}}$ - densidade geométrica, $\varphi_{\mathrm{s}}$ densidade específica do sólido

\section{RESULTADOS E DISCUSSÃO}

\section{Gesso $\beta-\left(\mathrm{CaSO}_{4} \cdot 0,5 \mathrm{H}_{2} \mathrm{O} /\right.$ Sulfato de cálcio hemihidratado)}

A matéria prima e as condições de produção do gesso influenciam seu tempo de pega. Impurezas contidas na matéria prima podem afetar o tempo de pega, acelerando-a por atuarem como núcleos de cristalização. Desta forma, torna-se fundamental determinar a sua pureza.

Na Fig. 3a é apresentada a microestrutura de partículas do gesso hemihidratado, na qual se observam cristais com formas lamelares, com superfície lisa e irregular. O material também apresenta elevada homogeneidade. A caracterização morfológica é importante, pois influencia nas propriedades da pasta e do gesso endurecido. Por exemplo, para se obter misturas de mesma consistência, o hemidrato $\alpha$ requer menos água de amassamento que o $\beta$.

A análise semiquantitativa do gesso, obtida através de análise EDS (Fig. 3b), apresenta valores dos elementos, em porcentagem em massa. Verifica-se a presença dos elementos químicos cálcio, enxofre e oxigênio, que se referem à

Tabela II - Análise semiquantitativa do gesso $\beta$ por FRX. [Table II - Semiquantitative analysis of gypsum $\beta$ by XRF.]

\begin{tabular}{cccc}
\hline Elemento & \% em massa & Elemento & \% em massa \\
\hline Cálcio (Ca) & 66,319 & Estrôncio (Sr) & 0,207 \\
Enxofre (S & 29,929 & Ferro (Fe) & 0,085 \\
Fósforo (P) & 3,463 & & \\
\hline
\end{tabular}



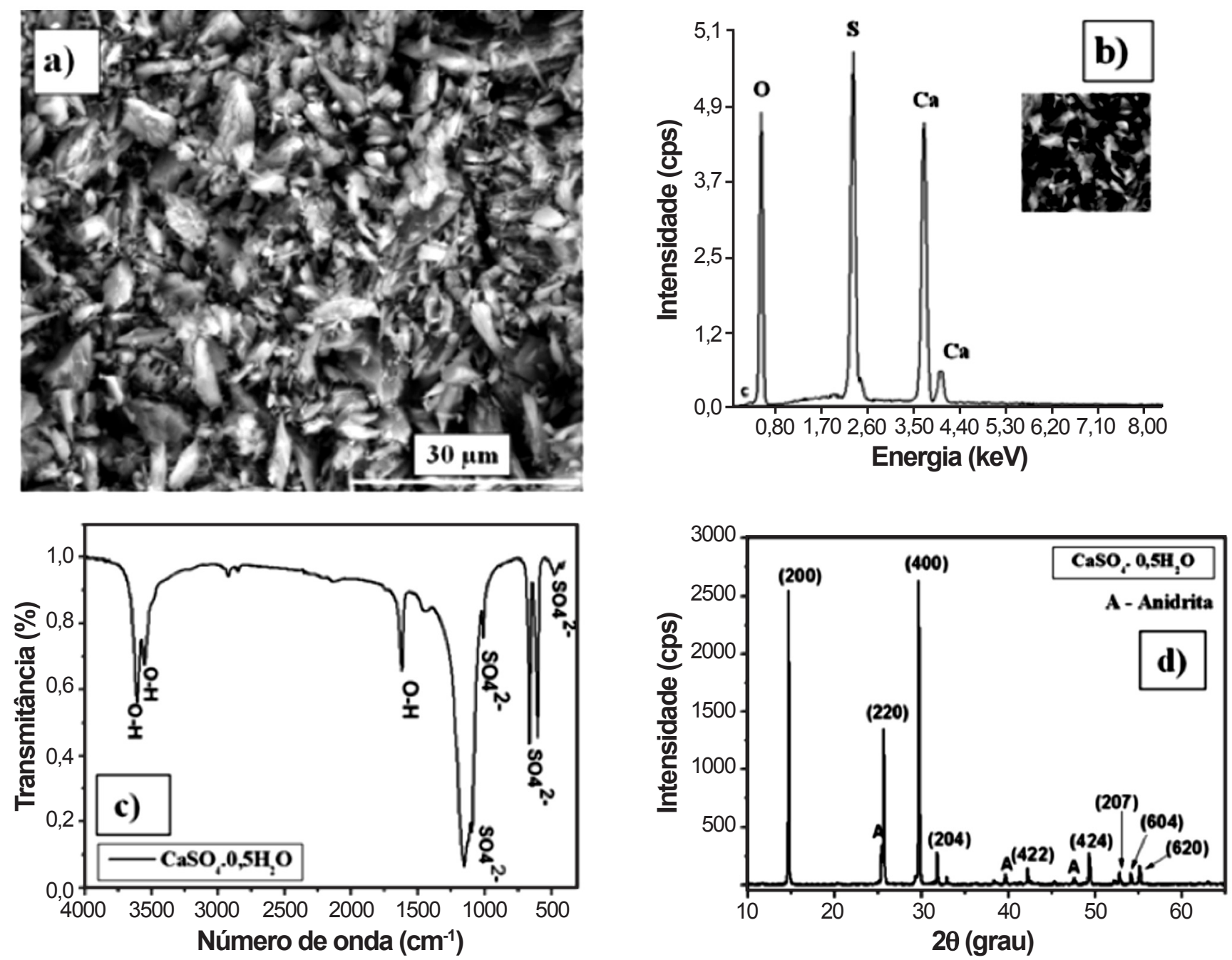

Figura 3: a) Micrografia obtida em microscopia eletrönica de varredura do pó de gesso hemihidratado; b) EDS correspondente à amostra $a$ ); c) Espectro de infravermelho e d) Difratograma de raios X do sulfato de cálcio hemihidratado.

[Figure 3: a) SEM micrograph of gypsum powder; b) EDS of the sample; c) Infrared spectrum and d) X-ray diffraction pattern of the hemihydrate of calcium sulphate.]

composição química do sulfato de cálcio e, portanto, confirma a elevada pureza da matéria prima. A pureza do hemihidrato foi também avaliada a partir da análise química quantitativa por espectrometria de fluorescência de raios X. A composição química do gesso $\beta$, Tabela II, indica a presença de pequenos traços de outros componentes, além do sulfato de cálcio, dentre os quais se encontram $\mathrm{P}, \mathrm{Sr}$ e Fe. No entanto, diante das frações mássicas atribuídas as impurezas presentes, pode-se observar a elevada pureza do minério.

A análise química do gesso foi complementada por meio da obtenção de um espectro de infravermelho do hemihidrato, Fig. 3c, com esta análise foi possível confirmar a presença dos seus grupos funcionais com bandas em 1625, $3556 \mathrm{e}$ $3612 \mathrm{~cm}^{-1}$ referentes ao grupo $(\mathrm{O}-\mathrm{H})$, estas são atribuídas às moléculas de água encontradas na estrutura do hemihidrato. Os grupos sulfato $\left(\mathrm{SO}_{4}^{2-}\right)$ também foram identificados em 591, 663, 1007 e $1132 \mathrm{~cm}^{-1}$. Estes resultados corroboram com as análises da fluroescencia de raios X e XEDS, onde confirmam a presença do enxofre sob a forma do grupo sulfato.

$\mathrm{O}$ difratograma de raios $\mathrm{X}$ do pó de gesso $\beta$ (Fig. 3d) demonstra que a amostra é composta essencialmente por hemidrato, pois foram identificados os picos carcterísticos de bassanita ( $\mathrm{B})\left(\beta-\mathrm{CaSO}_{4} \cdot 1 / 2 \mathrm{H}_{2} \mathrm{O}\right)$, e também foram detectadas picos correspondentes à anidrita $(\mathrm{A})\left(\mathrm{CaSO}_{4}\right)$. Os picos de maior intensidade são encontrados em $2 \theta=14,67^{\circ}, 25,72^{\circ}$ e $29,79^{\circ}$, correspondentes aos respectivos planos (200), (220) e (400). Picos de menor intensidade foram observados em $2 \theta=31,79^{\circ}, 42,24^{\circ}, 49,18^{\circ}, 52,92^{\circ}, 54,09^{\circ}$ e $55,11^{\circ}$, associados aos planos (204), (422), (424), (207), (604) e (620), respectivamente Todos esses picos são atribuídos a bassanita [9]. Alguns picos de mínima intensidade em $2 \theta=$ $25,33^{\circ}, 39,66^{\circ}$ e $47,58^{\circ}$ foram identificados no difratograma, estes são atribuídos à anidrita, material que consiste em um dos subprodutos da calcinação da gipsita. A anidrita apresenta três diferentes formas, anidrita III $\left(\mathrm{CaSO}_{4}\right.$. $\left.\xi \mathrm{H}_{2} \mathrm{O}\right)$, a anidrita $\mathrm{II}\left(\mathrm{CaSO}_{4}\right)$ e a anidrita $\mathrm{I}\left(\mathrm{CaSO}_{4}\right)$, estas dependem 
da faixa de temperatura empregada na calcinação. A 160 a $200{ }^{\circ} \mathrm{C}$ obtém-se a anidrita III, uma fase solúvel, porém instável, ávida por água, que se transforma em hemihidrato rapidamente. De 250 a $800{ }^{\circ} \mathrm{C}$ tem-se a anidrita II, insolúvel com velocidade de hidratação lenta, e acima de $800^{\circ} \mathrm{C}$ a fase é a anidrita I, que resulta em um produto de endurecimento lento $[7,9]$.

As dimensões e distribuição granulométrica do gesso foram determinadas por granulometria a laser. A determinação do tamanho das partículas é fundamental, pois influencia na cinética da reação. A taxa de hidratação aumenta com a diminuição do tamanho das partículas e conseqüente aumento da área específica do material.

A curva de distribuição granulométrica do material, Fig. 4, mostra uma banda principal com máximo entre $5 \mu \mathrm{m}$ e $9 \mu \mathrm{m}$. A faixa de valores encontrada para a dimensão das partículas varia entre $0,09 \mu \mathrm{m}$ e $351,5 \mu \mathrm{m}$, sendo cerca de $10 \%$ das partículas inferiores a $3,2 \mu \mathrm{m}, 50 \%$ inferiores a $12,1 \mu \mathrm{m}$ e $90 \%$ inferiores a $77,8 \mu \mathrm{m}$.

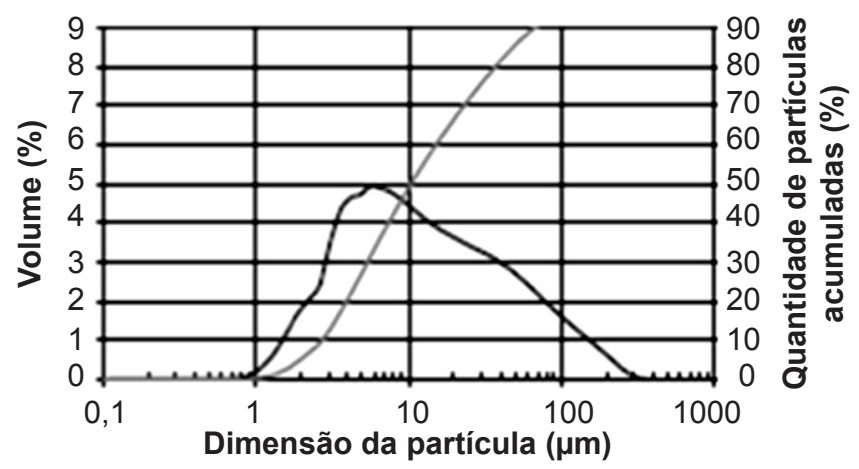

Figura 4: Curva de distribuição granulométrica do gesso. [Figure 4: Curve of particle size distribution of gypsum.]

A partir dos resultados apresentados nesta primeira etapa do trabalho, conclui-se que o gesso $\beta$ proveniente do polo gesseiro do Araripe apresenta uma morfologia com cristais lamelares, de superfície lisa e irregular. É constituído de partículas com dimensões variando entre $0,09 \mu \mathrm{m}$ e 351,5 $\mu \mathrm{m}$. Sendo composto basicamente pelos seguintes elementos: $\mathrm{Ca}, \mathrm{S}$ e O. O difratograma de raios X do pó de gesso mostra que a amostra é composta essencialmente por hemidrato, pois foram identificados os picos carcterísticos de bassanita (B) $\left(\beta-\mathrm{CaSO}_{4} \cdot 1 / 2 \mathrm{H}_{2} \mathrm{O}\right)$, e também foram detectadas picos correspondentes à anidrita $(\mathrm{A})\left(\mathrm{CaSO}_{4}\right)$.

Corpo sólido de gesso ( $\mathrm{CaSO}_{4} \cdot 2 \mathrm{H}_{2} \mathrm{O} /$ sulfato de cálcio dihidratado)

Com o objetivo de investigar o efeito da adição de água ao pó de gesso $\beta$, analisaram-se as diferentes razões em massa de água/gesso: 0,5, 0,6 e 0,7. As melhores relações foram selecionadas em função das propriedades do compósito, tais como o tempo de pega e as propriedades mecânicas. Analisou-se a influência do aumento da relação a/g na pasta de gesso através das micrografias apresentadas nas Figs. 5a, b e c.

A relação água/gesso $(\mathrm{a} / \mathrm{g})$ exerce influência na taxa de precipitação dos cristais de dihidrato. Os cristais de dihidrato crescem a partir de germes de cristalização ou núcleos. A quantidade de núcleos na solução vai influenciar a taxa de crescimento e o tamanho dos cristais. Quando os núcleos são numerosos, o crescimento é rápido e há formação de uma quantidade de pequenos cristais por unidade de volume da solução. Quando ocorre a formação de poucos núcleos o crescimento é lento, o que favorece a formação de cristais grandes. Microestruturas formadas de cristais grandes são menos resistentes (mais porosas) que as formadas por cristais pequenos (mais densas) [7]. Os cristais do dihidrato, Figs. $5 \mathrm{a}, 5 \mathrm{~b}$ e $5 \mathrm{c}$, têm, predominantemente, a forma de agulhas prismáticas, as quais consistem na morfologia padrão para o sulfato de cálcio dihidratado [10]. Além da
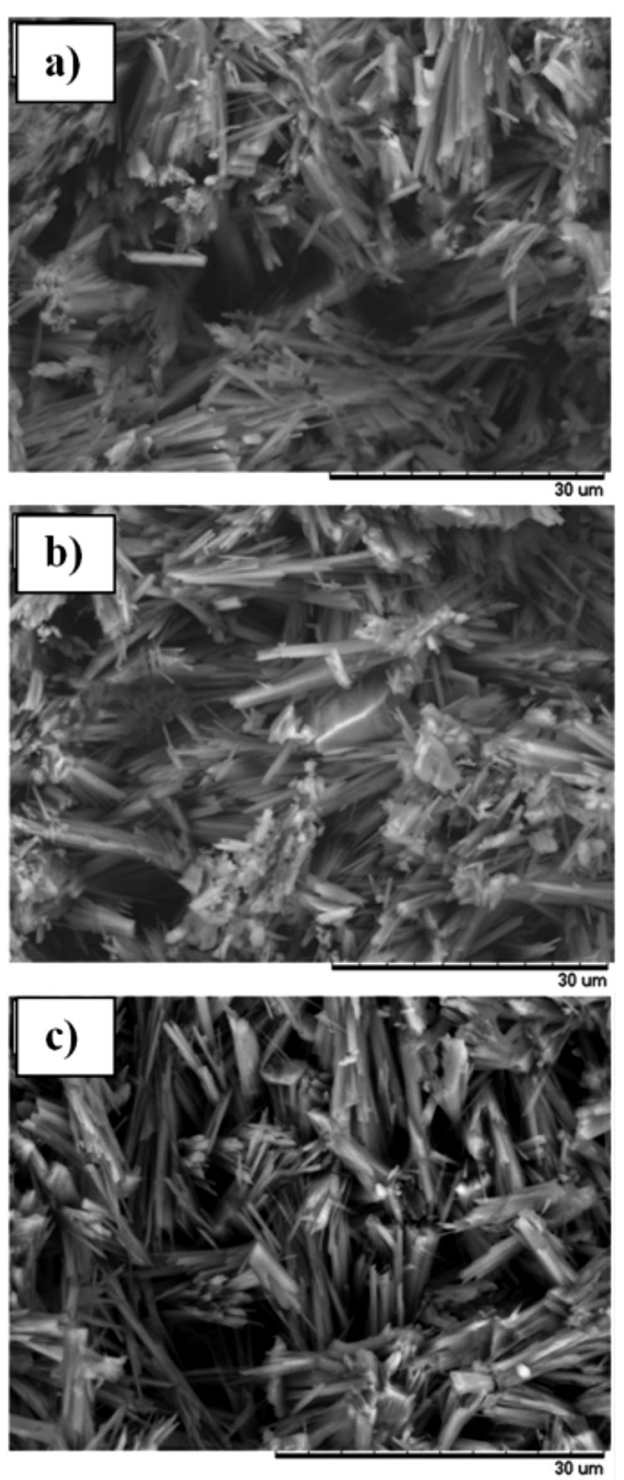

Figura 5: Micrografias obtidas em microscópio eletrônico de varredura de fratura do gesso dihidratatado, a) $\mathrm{a} / \mathrm{g}=0,5 ; \mathrm{b}$ ) $\mathrm{a} / \mathrm{g}=0,6$ e c) $\mathrm{a} / \mathrm{g}=0,7$. [Figure 5: SEM micrographs of dihydrate gypsum fracture, a) $w / g=0.5$;b) $w / g=0.6$ ec) $w / g=0.7$.] 
forma dos cristais, também se constata alta porosidade do material, principalmente com o aumento da razão a/g. Esta é proporcionada devido à água remanescente do processo de hidratação do pó de gesso, ocorrência que se deve a utilização de uma quantidade de água superior ao valor estequiométrico para hidratação do hemihidrato, com isso a quantidade de água que não reage com o pó, ocupa volume entre os cristais, e após a cura do dihidrato, a água evapora deixando vazios no material [11]. Em todas as micrografias observou-se um aspecto heterogêneo com aglomerados de diferentes tamanhos, e com a presença de poros de diferentes tamanhos distribuídos aleatoriamente.

A caracterização composicional e cristalográfica do gesso dihidratado foi feita por meio das técnicas de XEDS, FTIR e difração de raios X (Figs. 6a, 6b, 6c e 6d), respectivamente. A análise do XEDS (Fig. 6b) comprova a presença dos elementos constituintes das fases cristalinas correspondentes
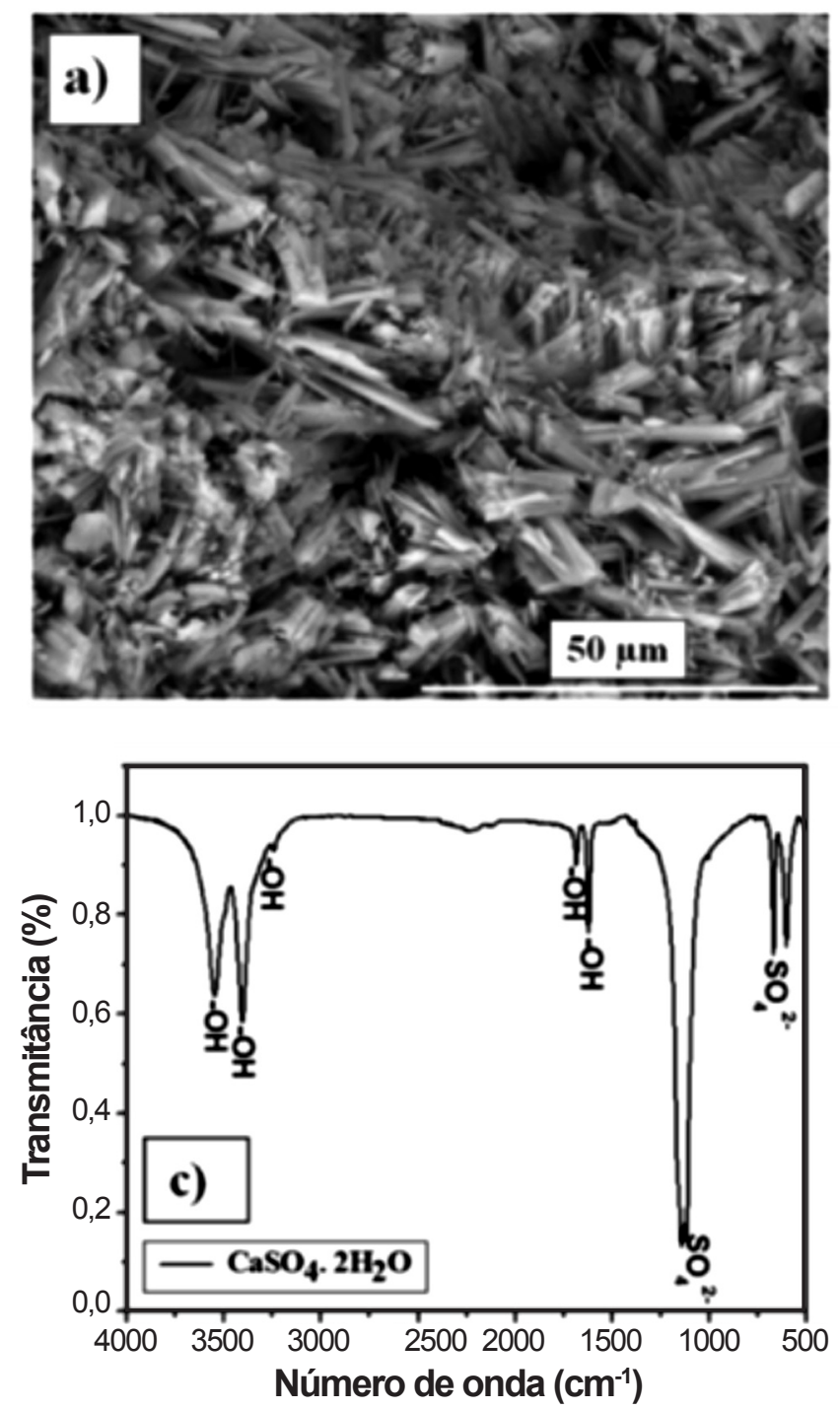

ao gesso $\beta$. Apresentando cálcio, oxigênio e enxofre, isto porque o material de partida é o mesmo, diferenciandose apenas por um acréscimo de água no material. Esta observação é complementada por meio do espectro de infravermelho, Fig. 6c. Neste, verifica-se a semelhança das bandas de absorção em relação às obtidas para o hemihidrato, Fig. 3c. Esta ocorrência se deve ao fato de que ambos os materiais apresentam basicamente a mesma composição química, sendo diferenciados apenas pela quantidade de água de hidratação, confirmada pela presença de uma banda em $1682 \mathrm{~cm}^{-1}$ associada ao grupo (-OH), que ocorre apenas para o dihidrato [12]. O difratograma de raios X da Fig. 6d confirma que a amostra é composta essencialmente pelo dihidrato, apresentando picos bastante intensos em $2 \theta=11,61^{\circ}, 20,69^{\circ}$ e $29,16^{\circ}$ correspondentes aos seus respectivos planos (020), (021) e (041). Picos de menor intensidade foram observados em $2 \theta=23,40^{\circ}, 28,16^{\circ}, 31,07^{\circ}$ e $33,34^{\circ}$, estes são associados
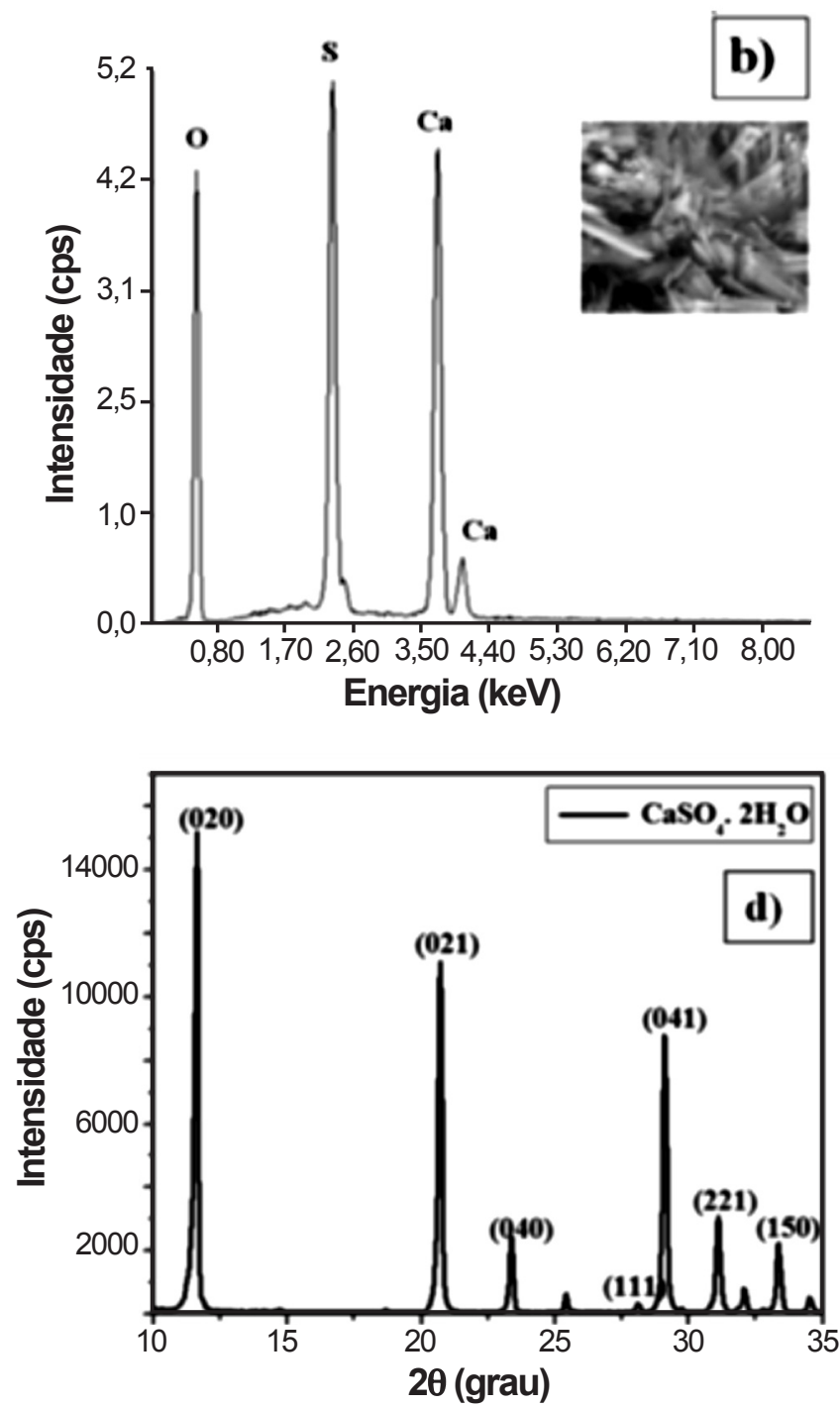

Figura 6: a) Micrografia obtida em microscópio eletrônico de varredura da fratura de gesso dihidratado; b) EDS da amostra; c) Espectro de infravermelho e d) Difratograma de raios X do sulfato de cálcio dihidratado.

[Figure 6: a) Micrograph of dihydrate gypsum fracture; b) EDS of the sample; c) Infrared spectrum and d) X-ray diffraction pattern of the calcium sulfate dihydrate.] 
aos planos (040), (111), (221),(150) respectivamente, também característicos do sulfato de cálcio.

Após o conhecimento da microestrutura característica do dihidrato, foram realizados ensaios para verificação da trabalhabilidade da pasta. Além disso, também foram observadas a porosidade e a resistência mecânica do material em função das diferentes razões a/g. O estudo da relação água/ gesso é o parâmetro de maior influência na cinética da reação de hidratação e, consequentemente, na pega do gesso. Quanto maior a quantidade de água de amassamento, maior o intervalo de tempo necessário para saturar a solução. Isto causa a ampliação do período de indução, retardando o início da precipitação dos cristais de dihidrato e, por conseguinte, aumenta o tempo de pega. Assim sendo, quanto maior a relação água/gesso, menor a taxa da reação e maior o tempo de pega. A relação água/gesso (a/g) exerce influência na taxa de precipitação dos cristais de dihidrato e, por conseguinte, na variação da consistência da pasta ao longo do tempo, que é o fator determinante para
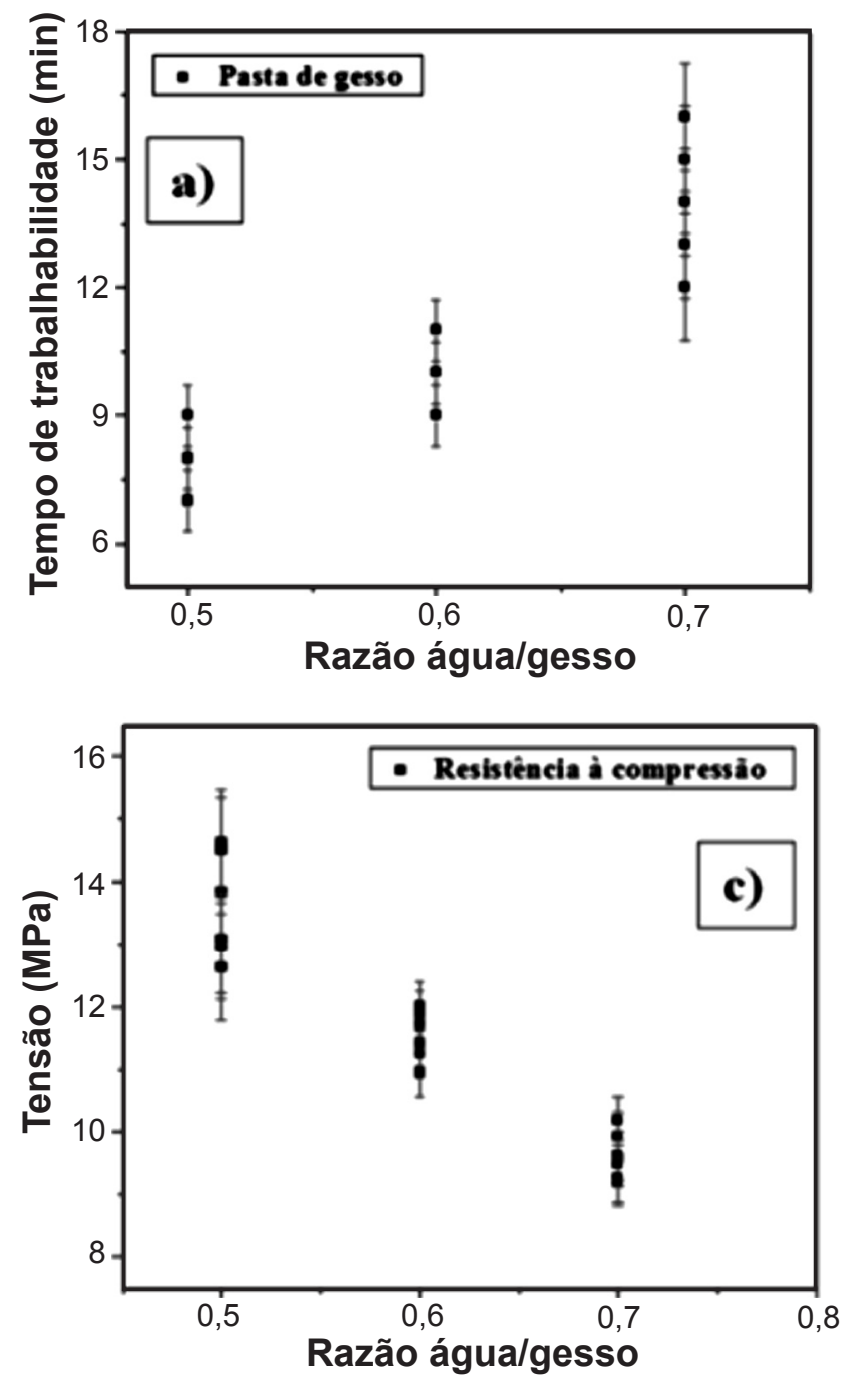

sua aplicação.

A partir da Fig. 7a pode-se observar o aumento do tempo de trabalhabilidade das pastas de gesso, de acordo com a proporção de água utilizada no seu preparo. Comparandose os dados apresentados, constata-se um tempo variando de $8 \pm 0,73$ a $14 \pm 1,26$ min, para uma proporção de água de 0,5 a 0,7 , respectivamente. O mesmo comportamento foi observado para a porosidade geométrica dos blocos após 14 dias de cura, Fig. 7b. De acordo com a literatura [13], a água consiste no fator de maior influência no processo de dissolução-cristalização do gesso. O mecanismo de cristalização inicia-se pelo processo de nucleação. A porosidade do dihidrato está diretamente relacionada à velocidade de nucleação, pois a formação de grandes aglomerados com formas irregulares proporciona uma menor compactação dos cristais. Quanto maior a quantidade de água utilizada na hidratação do pó, maior é a quantidade de poros deixados após a cura do bloco [11, 13]. Este resultado
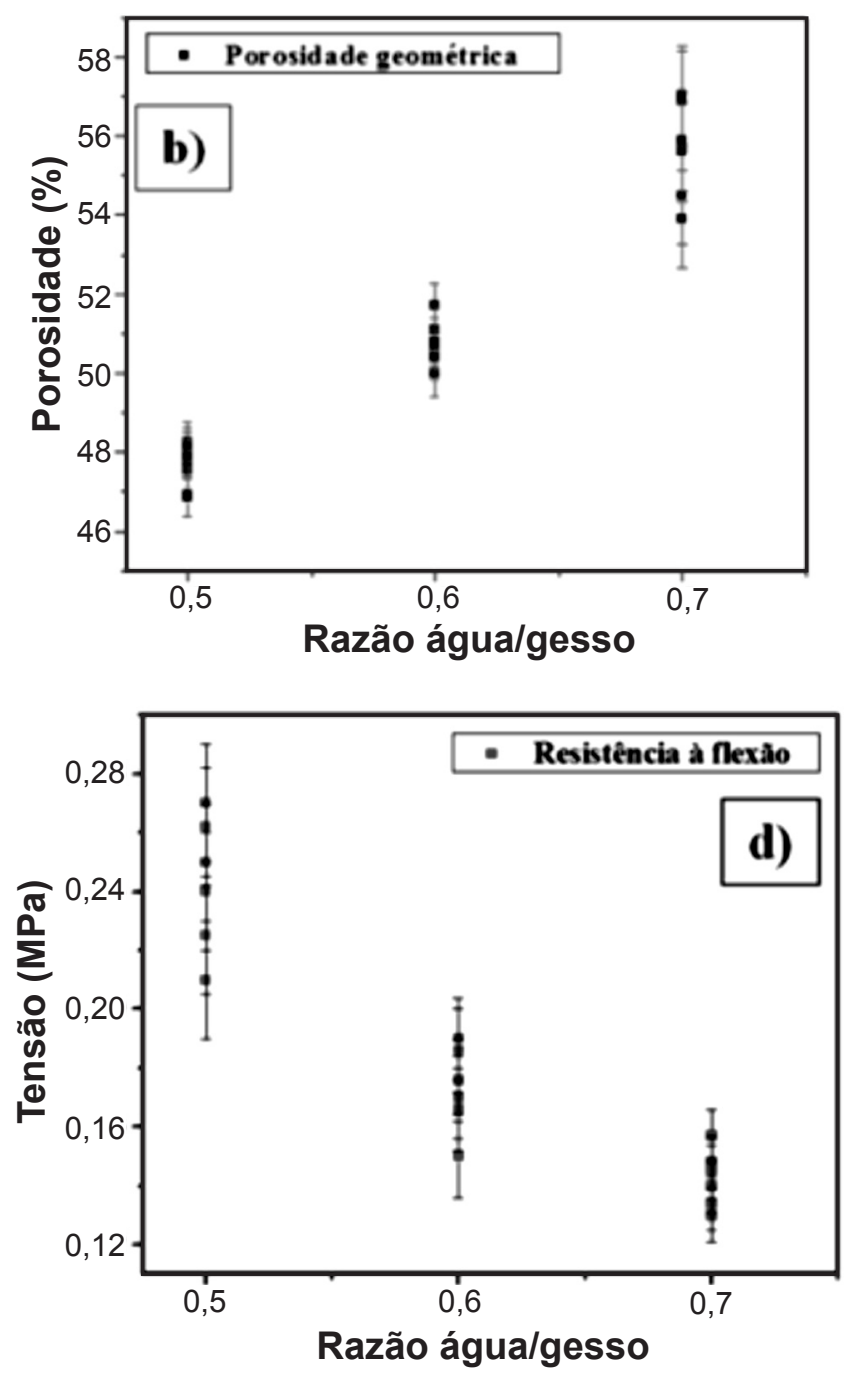

Figura 7: a) Tempo de trabalhabilidade da pasta de gesso; b) Porosidade geométrica; c) Resistência à compressão e d) Resistência à flexão, correspondentes a diferentes razões água/gesso, variando de 0,5 a 0,7 .

[Figure 7: a) Workability time of gypsum slurry; b) Geometric porosity; c) Compressive strength and d) Flexural, corresponding to different reasons water/gypsum, varying 0.5 to 0.7 .] 
corrobora com as micrografias apresentadas nas Figs. 5a, $5 \mathrm{~b}$ e $5 \mathrm{c}$. Além da razão a/g, a forma do cristal também influencia nas propriedades mecânicas da pasta endurecida. Os cristais do dihidrato têm, predominantemente, a forma de agulhas e apresentam um bom intertravamento. A resistência mecânica ( $\boldsymbol{\sigma c}$ e $\boldsymbol{\sigma f})$ do sulfato de cálcio dihidratado apresenta uma relação inversamente proporcional com a porosidade dos corpos sólidos, Figs. 7c e 7d. Desta forma, as resistências mecânicas à $\boldsymbol{\sigma c}$ e $\boldsymbol{\sigma} \boldsymbol{f}$ caem com o aumento da proporção de água e consequentemente com o aumento da porosidade dos corpos. Estes resultados corroboram com diversos trabalhos encontrados na literatura $[11,14,15]$. Pode-se constatar também que a resistência à compressão obtida para o gesso caracterizado neste trabalho apresentou valor superior ao mínimo exigido pela a NBR 13207 que é de 8,4 MPa [16].

Pode-se considerar que o gesso $\beta$ se trata de uma matéria prima de elevado potencial, pois como constatado por meio das análises, ele se apresenta com composição de sulfato de cálcio praticamente puro, e, portanto, pode ser utilizado em novas aplicações de maior valor agregado, como por exemplo, uma possível extração do metal cálcio, metal de alto valor comercial, a partir do gesso do Araripe. Verificase uma necessidade de estudos que tratem do melhoramento da resistência mecânica do gesso dihidratado, pois este, apesar da boa aparência oferecida quando empregado na construção civil, possui resistência muito baixa, e por isso, tem sua aplicação limitada.

\section{CONCLUSÃO}

Com base nas diversas análises realizadas na matéria prima gerada pela calcinação da gipsita extraída do pólo gesseiro do Araripe, o gesso $\beta$, foi possível confirmar sua elevada pureza. Diante do conhecimento da morfologia apresentada pelo gesso hemi e dihidratado, também se constataram a forma dos seus cristais e sua relação entre a proporção de água e respectiva resistência mecânica suportada pelo material. Características químicas, como grupos funcionais presentes no gesso também foram observados por meio de Espectros de Infravermelho, assim como impurezas presentes no gesso $\beta$, resultantes dos minérios da região, que foram detectados por fluorescência de raios X em mínima quantidade. Com base nas informações adquiridas neste trabalho de caracterização, pretende-se continuar a realização de estudos para um melhor aproveitamento das características apresentadas pela matéria prima, bem como buscar soluções para as fragilidades observadas, como por exemplo, a baixa resistência mecânica dos corpos sólidos de gesso.

\section{AGRADECIMENTOS}

À CAPES, pelo suporte financeiro, e à Indústria Gesso Mineral pelo fornecimento do gesso.

\section{REFERÊNCIAS}

[1] A. C. P. L. Lira, A. J. R. Amaral, J. O. C. Dantas, J. R. A. Dantas, Gipsita, Balanço Mineral Brasileiro, DNPM/PE (2001).

[2] H. F. Dantas, R. A. S. Mendes, R. D. Pinho, L. E. B. Soledade, C. A. Paskocimas, B. B. Lira, M. O. E. Schwartz, A. G. Souza, M. G. Santos, J. Thermal Anal. Calorim. 87 (2007) 691-695.

[3] C. A. M. Baltar, F. F. Bastos, A. B. Luz, Gipsita, Comun. Tec., Ed. Rochas \& Minerais Industriais: Usos e Especificações, Rio de Janeiro, RJ (2005) 449-470.

[4] D. B. Jorgensen, Industrial Minerals and Rocks, Ed. D. D. Carr, Soc. Mining, Metallurgy Exploration, Inc., Littleton, Colorado, EUA (1994).

[5] A. A. Barbosa, Diss. Mestrado Ciência dos Materiais, Universidade Federal do Vale do S. Francisco, Juazeiro, BA (2012).

[6] Associação Brasileira de Normas Técnicas - NBR 13207, Método de ensaio, NBR 12128, Rio de Janeiro, RJ (1994).

[7] R. P. N. Antunes, Diss. Mestrado Engenharia, Universidade de S. Paulo, S. Paulo, SP (1999).

[8] M. M. Akiyoshi, A. P. Silva, M. G. Silva, V. C. Pandolfelli, Cerâmica 47, 303 (2001) 163-169.

[9] M. G. S. Silva, Diss. Mestrado Ciência e Engenharia de Materiais, Universidade Federal de Sergipe, S. Cristovão, SE (2010).

[10] P. Reynaud, M. Saâdaqui, S. Meille, G. Fantozzi, Mater. Sci. Eng. 442 (2006) 500-503.

[11] W. M. Kanno, Tese Dr. Ciência e Engenharia de Materiais, Universidade de S. Paulo, S. Carlos, SP (2009).

[12] K. P. Mandal, T. K. Mandal, Cem. Concr. Res. 32 (2002) 313-316.

[13] J. Karni, E. Karni. Mater. Structure 28 (1995) 92-100.

[14] M. Rossler, I. Odler, Zement-Kalk-Gips 2 (1989) 96100.

[15] A. M. N. Dias, M. A. Cincoto, Bol. Tec. Escola Politécnica da USP, S. Paulo, SP (1995).

[16] Associação Brasileira de Normas Técnicas - NBR 13207, Método de ensaio, NBR 12129, Rio de Janeiro, RJ (1994).

(Rec. 07/02/2014, Rev. 04/09/2014, Ac. 17/09/2014) 